Journal for ImmunoTherapy of Cancer

\title{
Extending the conversation over the immune-related hepatotoxicity: author response to Dr. Gauci et al
}

\author{
Dimitrios C Ziogas, Helen Gogas
}

To cite: Ziogas DC, Gogas H. Extending the conversation over the immune-related hepatotoxicity: author response to Dr. Gauci et al. Journal for ImmunoTherapy of Cancer 2021;9:e002391. doi:10.1136/ jitc-2021-002391

Accepted 17 February 2021

\section{SLinked}

http://dx.doi.org/10.1136/ jitc-2020-001322

Check for updates

(C) Author(s) (or their employer(s)) 2021. Re-use permitted under CC BY-NC. No commercial re-use. See rights and permissions. Published by BMJ.

First Department of Medicine, National and Kapodistrian University of Athens School of Medicine, Athens, Greece

Correspondence to Professor Helen Gogas; helgogas@gmail.com

\begin{abstract}
Immune-related hepatotoxicity $(\mathrm{IRH})$ remains the subject of many immune-oncology debates due to its challenging diagnosis and management. Although it is currently defined by the restrictive Common Terminology Criteria for Adverse Events (CTCAE), the term of IRH covers a wide range of liver pathologies, including hepatitic, cholangitic, mixed, steatotic and nonspecific patterns of injury. Even when liver biopsy is performed, the recognized histopathological findings cannot predict the response to steroids or the need for secondary immunosuppression, and usually do not significantly modify the suggested empirical treatment of IRH. Beyond the CTCAE grading, a more comprehensive assessment of IRH severity, including laboratory biomarkers and clinical features, should be developed and a more patient-oriented management should be established by additional randomized evidence, incorporating hepatology and immune-oncology experience.
\end{abstract}

Following our recent publication on immunerelated hepatotoxicity (IRH) under the title "When steroids are not enough in immunerelated hepatitis: current clinical challenges discussed on the basis of a case report", 1 we read with interest the targeted comments by Dr. Gauci et al and will try to further discuss the concerns they have raised, in order to contribute to the discussion of this hot topic. Since immune-checkpoint inhibitors (ICPIs) have become a commonplace in the treatment of many hematological and solid malignancies and their use will continue to grow in the coming years, being currently under investigation in adjuvant and neoadjuvant settings, the oncological community has shifted its focus on the ICPI-induced toxicities. Currently, IRH remains poorly characterized, including many overlapping immune-mediated liver pathologies with variable severity, different nature, and unspecified management.

\section{HOW TO MEASURE THE SEVERITY OF IRH?}

It is particularly true that the existing Common Terminology Criteria for Adverse
Events (CTCAE) could not evaluate the severity of IRH with accuracy and usually overestimated it. The CTCAE classification was created for patients on chemotherapy and a quantitative increase in liver functions tests (LFTs) could not work as a sole marker of severity in cases with immune-mediated liver injury. Indeed, the grading of transaminase elevation was not found to be associated with the histologic extent of liver damage, in patients with IRH $^{2}$ or in those with autoimmune hepatitis. ${ }^{3}$ In addition, after the recent approval of atezolizumab plus bevacizumab in first-line treatment of advanced hepatocellular carcinoma, ${ }^{45}$ more patients with liver decompensation due to viral hepatitis are currently exposed to ICPIs, and their risk of further immune-related liver deterioration should be more precisely assessed. ${ }^{6}$ In these vulnerable populations with pre-existing impaired LFTs due to chronic viral disease or cirrhosis, the insufficiency of CTCAE system to describe the liver functionality over a period of time, regardless of transaminases elevation, motivated the hepatologists to introduce the Child-Pugh score (eg, bilirubin level, albumin, prothrombin time (PT), encephalopathy, ascites). At this point, we should note that the classification of IRH severity needs to be revised to incorporate the clinical expertise of hepatologists, and more clinical and biological parameters should be included to provide a corresponding picture of liver status. Recently, Gauci $e t a l^{7}$ and De Martin et $a l^{8}$ agreed that PT and bilirubin level, two parameters of Child-Pugh score, should be examined, in addition to transaminases, to assess IRH grading while alkaline phosphatase (ALP) has been also suggested as an independent classifier of hepatotoxicity, indicating biliary obstruction or inflammation. ${ }^{910}$ In our case, all the aforementioned features (eg, PT: 13.5 s, AST: $1108 \mathrm{U} / \mathrm{L}$, ALT: $1252 \mathrm{U} / \mathrm{L}$, ALP: $328 \mathrm{U} / \mathrm{L}$, GGT: $621 \mathrm{U} / \mathrm{L}$, Bil: $6.7 \mathrm{mg} / \mathrm{dL}$ ) and further important 
inflammatory clinical and laboratory biomarkers, not yet associated with the IRH severity (eg, fever, increased CRP and high white blood cell count), confirmed the development of a severe immune-mediated inflammatory liver reaction. A specific grading system for IRH, including laboratory biomarkers (eg, AST, ALT, ALP, Bil, albumin, PT or unexplained lactic acidosis) and clinical features (pruritus, jaundice, petechiae, hepatic encephalopathy, ascites or other symptoms of liver disease), should be developed encompassing the hepatology and immuneoncology experience so far.

\section{HOW MANY LIVER PATHOLOGIES SHOULD BE INCLUDED IN DIFFERENTIAL DIAGNOSIS OF IRH? CAN A LIVER BIOPSY HELP?}

A further concern is that the existing CTCAE grading can simplify many diverse pathologies while immunotherapy can simultaneously lead to a multi-level hepatic (and extrahepatic) inflammation with overlapping histopathologic features. In an interesting analysis of liver biopsies from patients with IRH, Cohen et $a l^{2}$ tried to evaluate the pattern of liver inflammation and whether the specific pattern of liver injury correlates with LFT abnormalities, imaging findings and responsiveness to steroids. The pattern of ICPI-induced liver injury depends on the type of immunotherapy, the dose and the baseline liver status, and it may be hepatitic, cholestatic/cholangitic, mixed, steatotic (resembling fatty liver) or appear as mild non-specific changes. ${ }^{2}$ Liver biopsies in cases with severe IRH revealed varying degrees of lobular hepatitis with numerous histiocytes, endothelialitis, loose or wellformed granulomas, fibrin ring granulomas, and varying degrees of portal and periportal inflammation. ${ }^{2} 31112$ Several patients with IRH may develop predominantly biliary and/or peribiliary inflammation (cholangitic pattern), or may have histological findings of both lobular and ductal inflammation (mixed pattern). In these cases, when a liver biopsy is performed, immunostaining usually reveals portal (and maybe concurrent lobular) infiltrates by $\mathrm{CD} 8^{+}$T-lymphocytes ${ }^{13}$ with highly expressed granzyme $\mathrm{B}$, a marker of $\mathrm{T} \mathrm{CD} 8^{+}$activation. The cholangitic pattern of immune-related inflammation is more likely to have clinical signs of obstruction (eg, jaundice), and imaging findings such as bile duct dilatation or narrowing. ${ }^{1214-17}$ Notably, Doherty et $a l^{18}$ described three rare cases with ICPI-induced steroid-resistant hepatotoxicity, where the biliary tract was the main target of hepatotoxicity, and they documented a wide spectrum of ductal damage reaching up to vanishing bile duct syndrome. Despite the prolonged and severe course of biliary inflammation, all these three cases showed gradual improvement in LFTs after commencing steroids, while 2 of them required additional immunosuppression to recover. ${ }^{18}$ Recently, Onoyama $e t a l^{13}$ attempted to further distinguish this immune-related cholangitic pattern by focusing on anti-PD-1 treated cases with sclerosing cholangitis histopathology. The authors subclassified immune-related cholangitis in intrahepatic type (multiple stenoses in the intrahepatic bile duct, without extrahepatic biliary hypertrophy), extrahepatic type (diffuse extrahepatic biliary hypertrophy without biliary stenosis) and diffuse type (diffuse biliary tract hypertrophy with multiple stenoses of the intrahepatic and extrahepatic bile ducts). The clinical implications of this immune-related cholangitis classification were uncertain since standard management was followed with a response rate to corticosteroids that was extremely low (11.5\%, one case in extrahepatic and two cases in the intrahepatic type).

Liver biopsy is mandatory to detect the exact histological type of liver injury in cases with IRH, but it is not a universal approach in any case of grade $\geq 3$ IRH. Even Gauci $e t a l$ in their last publication performed liver biopsy in only 7 of the 21 patients with severe IRH (10 with grade 3 and 11 with grade 4$)$. Notably, this biopsy did not change the subsequent management and all but one biopsied patient received steroids, while six of these patients had already confirmed the severity of their liver injury, based only on their biological biomarkers (eg, increase of bilirubin and/or prolongation of PT). In our case, we decided not to perform a liver biopsy because of hemorrhagic risk, yet all other differential diagnoses, such as melanoma infiltration, cirrhosis, autoimmune or viral hepatitis, were ruled out by imaging and blood testing. The main disadvantage of not performing a biopsy in our case was that we could not define the pattern of IRH. However, even knowing the histological type of immunerelated liver injury, our management of IRH would probably not be at all different. According to Cohen et al, the pattern of liver inflammation, degree of lobular injury, or presence of granulomas or endothelitis does not predict response to steroids or the need for secondary immunosuppression. In support, Cheung et al diagnosed 21 cases with IRH among 453 immunotherapy-treated patients with cancer and managed them empirically without liver biopsy. ${ }^{19}$

\section{NECESSITY OF STEROIDS IN SEVERE IRH}

Currently, the expert oncology societies, ESMO, SITC and ASCO, for patients with immune-related grade 3 or 4 elevation of transaminases, with or without concurrent increase of bilirubin, suggest immediate initiation of steroids at $0.5-1 \mathrm{mg} / \mathrm{kg} /$ day. ${ }^{20-22}$ These therapeutic guidelines that were also followed in our case with IRH are mainly empirical recommendations as no clinical trials have been designed to support the need of steroids in the treatment of ir-hepatotoxicity or another ir-adverse event, in general. In contrast, several cases with IRH are reported, which spontaneously overcome their grade $\geq 3$ IRH. In the study of De Martin et al, 6 of 16 patients (38\%) resolve their IRH without receiving any corticosteroid therapy while no severe increase in LFTs was observed in two of them after an immunotherapy rechallenge. ${ }^{23}$ Moreover, De Martin et al proposed the administration of ursodeoxycholic acid alone as an initial approach in cases with minimal/no elevation of transaminases while steroids 
should be added if LFTs do not improve. ${ }^{23}$ In another paper by Gauci et al, half of patients with melanoma who experienced IRH over anti-PD-1 and/or CTLA-4 treatment resolved their ir-hepatic $\mathrm{AE}$ with no steroids and no second-line immunosuppression. ${ }^{24}$ It is worth noting that the administration of steroids did not significantly shorten the time to IRH resolution. ${ }^{24}$ Data presented in the pharmaceutical summary of the approved combination of nivolumab/ipilimumab with a higher incidence of IRH demonstrate that less than half of patients with melanoma $(46 \%)$ will require high-dose corticosteroid (eg, at least $40 \mathrm{mg}$ prednisone daily or equivalents) to resolve their immune-mediated liver toxicity.

There are no data to support that the histological evaluation of liver biopsy could drive the need of steroids in the treatment of severe IRH. Even in cases with cholangitic pattern where steroids seem not to be as efficient as in lobular injury, Izumi et al recognized 4 patients with nivolumab-induced cholangitis among 59 cases and treated them with corticosteroid alone $(\mathrm{n}=2)$ or in combination with MMF $(n=2)$, resulting in improvements in 3 of them. ${ }^{25}$ In addition to a comprehensive assessment of IRH severity, a more personalized management is also required, where liver biopsy should be suggested only in cases with significant diagnostic uncertainty and systemic steroids could be eventually avoided, depending on the severity of liver injury.

\section{WHEN STEROIDS ARE NOT ENOUGH}

In the rare conditions of steroid-resistant IRH, the introduction of further immunosuppression should be a multidisciplinary well-balanced decision after the monitoring of biological liver parameters during the first 5-7 days of steroids. In our presented case, we followed these recommendations and added MMF 5 days after initiation of steroids and 2 days after maximization of their dose, in order to stop ongoing severe liver deterioration. Reviewing the increasing literature about ir-hepatic AEs, we recognized only a few cases that required MMF to overcome IRH after failure of corticosteroids and even fewer cases that required further immunosuppressive agents in refractory cases to corticosteroids and MMF. Actually, Miller et al estimated that among the 433 patients with cancer who experienced any grade IRH, 67 required steroids, 10 had IRH recurrence after steroid tapering, and only 2 patients had persistent liver dysfunction and required $\mathrm{MMF}^{26}$; in the study of Cheung et al, only 3 out of 21 cases with IRH required a third-line immunosuppressant beyond steroids and $\mathrm{MMF}^{19}$ while Gauci et al presented the most positive safety data with none of the patients requiring second-line agent after steroids. Motivated by our case report, we sorted all immunosuppressive options for severe IRH and proposed a therapeutic algorithm for resistant cases to steroids, including the discrepancies between oncology experts' societies. In agreement to Gauci $e t a l$, we concluded that both MMF and tacrolimus have strong anti-lymphocyte activity, proven in the setting of liver transplantation, and were reasonably among the first agents examined in steroid-refractory cases with IRH. However, both drugs induce suppression of lymphocyte-driven tumor surveillance and may lead to rapid cancer progression, as happened in the presented patient. In general, at any stage of IRH treatment, there is no sufficient prospective evidence to support one immunosuppressive therapeutic approach over another.

\section{WHAT ABOUT IMMUNOTHERAPY RESUMPTION?}

According to ESMO, SITC and ASCO guidelines, for patients with grade $\geq 3$ elevation of transaminases, with or without concurrent bilirubin increase, ICPIs should be permanently discontinued. ${ }^{20-22}$ There are many studies that question this proposed strategy and support the resumption of immunotherapy without a great risk of hepatotoxicity recurrence. ${ }^{1923242728}$ Gauci et al cited the study of De Martin et al where immunotherapy was reintroduced in 3 out of 16 patients with IRH, without recurrence of liver dysfunction, and presented their results where immunotherapy was resumed for 8 patients with no case of IRH relapse, and no need for low-dose steroids to prevent recurrence. ${ }^{23}$ According to the most recent retrospective analysis of 31 patients with melanoma with IRH who underwent ICPI rechallenge, 6 required ICPI discontinuation due to severe ir-AE of any type and 4 of these 6 cases developed recurrent IRH. ${ }^{29}$ The rapid rate of resolution of transaminase elevations may also give some more points in a resumption decision. ${ }^{10}$ In all cases of ICPI rechallenge, it is important to note that close monitoring is critically important as liver injury may recur rapidly and may be difficult to control. Moving out-ofthe-box in patients with limited therapeutic options and balancing the benefit-risk ratio in each individual case, an ICPI (of the same or other class) could be resumed in patients with melanoma who have recovered from grade 3 or 4 IRH with a modest risk of toxicity relapse. This can also be a potential approach in our patient if melanoma progresses with distant metastases, but it remains unclear whether ICPI retreatment improves clinical outcomes.

In the evolving immunotherapy landscape, the issue of IRH should be further examined and randomized evidence-based guidelines regarding its diagnosis and management should be developed.

Contributors DCZ and HG involved in the literature review and wrote the manuscript. Both authors edited, read and approved the final manuscript.

Funding The authors have not declared a specific grant for this research from any funding agency in the public, commercial or not-for-profit sectors.

Competing interests $\mathrm{HG}$ has received grants and personal fees by Roche, BMS, MSD, Novartis and personal fees by Amgen and Pierre Fabre, outside the submitted work.

Patient consent for publication Not required.

Provenance and peer review Commissioned; internally peer reviewed.

Open access This is an open access article distributed in accordance with the Creative Commons Attribution Non Commercial (CC BY-NC 4.0) license, which permits others to distribute, remix, adapt, build upon this work non-commercially, and license their derivative works on different terms, provided the original work is 
properly cited, appropriate credit is given, any changes made indicated, and the use is non-commercial. See http://creativecommons.org/licenses/by-nc/4.0/.

\section{REFERENCES}

1 Ziogas DC, Gkoufa A, Cholongitas E, et al. When steroids are not enough in immune-related hepatitis: current clinical challenges discussed on the basis of a case report. $J$ Immunother Cancer 2020;8:e001322.

2 Cohen JV, Dougan M, Zubiri L. Liver biopsy findings in patients on immune checkpoint inhibitors. Mod Pathol 2020;34:1-12.

3 Everett J, Srivastava A, Misdraji J. Fibrin ring granulomas in checkpoint inhibitor-induced hepatitis. Am J Surg Pathol 2017:41:134-7.

4 Finn RS, Qin S, Ikeda M, et al. Atezolizumab plus bevacizumab in unresectable hepatocellular carcinoma. $N$ Engl J Med 2020;382:1894-905.

5 Gordan JD, Kennedy EB, Abou-Alfa GK, et al. Systemic therapy for advanced hepatocellular carcinoma: ASCO guideline. J Clin Oncol 2020;38:4317-45.

6 Ziogas DC, Kostantinou F, Cholongitas E, et al. Reconsidering the management of patients with cancer with viral hepatitis in the era of immunotherapy. J Immunother Cancer 2020;8:e000943.

7 Gauci M-L, Baroudjian B, Bédérède U, et al. Severe immunerelated hepatitis induced by immune checkpoint inhibitors: clinical features and management proposal. Clin Res Hepatol Gastroenterol 2020:101491.

8 De Martin E, Michot J-M, Rosmorduc O, et al. Liver toxicity as a limiting factor to the increasing use of immune checkpoint inhibitors. JHEP Rep 2020;2:100170.

9 Eigentler TK, Hassel JC, Berking C, et al. Diagnosis, monitoring and management of immune-related adverse drug reactions of anti-PD-1 antibody therapy. Cancer Treat Rev 2016;45:7-18.

10 Reynolds K, Thomas M, Dougan M. Diagnosis and management of hepatitis in patients on checkpoint blockade. Oncologist 2018;23:991-7.

11 Johncilla M, Misdraji J, Pratt DS, et al. Ipilimumab-associated hepatitis: clinicopathologic characterization in a series of 11 cases. Am J Surg Pathol 2015;39:1075-84.

12 Zen $\mathrm{Y}$, Chen $\mathrm{Y}-\mathrm{Y}$, Jeng $\mathrm{Y}-\mathrm{M}$, et al. Immune-related adverse reactions in the hepatobiliary system: second-generation check-point inhibitors highlight diverse histological changes. Histopathology 2020;76:470-80.

13 Onoyama T, Takeda Y, Yamashita T, et al. Programmed cell death-1 inhibitor-related sclerosing cholangitis: a systematic review. World $J$ Gastroenterol 2020;26:353-65.

14 Hamoir C, de Vos M, Clinckart F, et al. Hepatobiliary and pancreatic: nivolumab-related cholangiopathy. J Gastroenterol Hepatol 2018;33:1695.
15 Gelsomino F, Vitale G, Ardizzoni A. A case of nivolumab-related cholangitis and literature review: how to look for the right tools for a correct diagnosis of this rare immune-related adverse event. Invest New Drugs 2018;36:144-6.

16 Gelsomino F, Vitale G, D'Errico A, et al. Nivolumab-induced cholangitic liver disease: a novel form of serious liver injury. Ann Oncol 2017;28:671-2.

17 Kawakami H, Tanizaki J, Tanaka K, et al. Imaging and clinicopathological features of nivolumab-related cholangitis in patients with non-small cell lung cancer. Invest New Drugs 2017;35:529-36.

18 Doherty GJ, Duckworth AM, Davies SE, et al. Severe steroid-resistant anti-PD1 T-cell checkpoint inhibitor-induced hepatotoxicity driven by biliary injury. ESMO Open 2017;2:e000268.

19 Cheung V, Gupta T, Payne M, et al. Immunotherapy-related hepatitis: real-world experience from a tertiary centre. Frontline Gastroenterol 2019;10:364-71.

20 Haanen JBAG, Carbonnel F, Robert C, et al. Management of toxicities from immunotherapy: ESMO Clinical Practice Guidelines for diagnosis, treatment and follow-up. Ann Oncol 2018;29:iv264-6.

21 Brahmer JR, Lacchetti C, Schneider BJ, et al. Management of immune-related adverse events in patients treated with immune checkpoint inhibitor therapy: American Society of Clinical Oncology Clinical Practice Guideline. J Clin Oncol 2018;36:1714-68.

22 Puzanov I, Diab A, Abdallah K, et al. Managing toxicities associated with immune checkpoint inhibitors: consensus recommendations from the Society for Immunotherapy of Cancer (SITC) Toxicity Management Working Group. J Immunother Cancer 2017;5:95.

23 De Martin E, Michot J-M, Papouin B, et al. Characterization of liver injury induced by cancer immunotherapy using immune checkpoint inhibitors. J Hepatol 2018;68:1181-90.

24 Gauci M-L, Baroudjian B, Zeboulon C, et al. Immune-related hepatitis with immunotherapy: are corticosteroids always needed? J Hepatol 2018;69:548-50.

25 Izumi H, Kodani M, Kurai J, et al. Nivolumab-induced cholangitis in patients with non-small cell lung cancer: case series and a review of literature. Mol Clin Oncol 2019;11:439-46.

26 Miller ED, Abu-Sbeih H, Styskel B, et al. Clinical characteristics and adverse impact of hepatotoxicity due to immune checkpoint inhibitors. Am J Gastroenterol 2020;115:251-61.

27 Pollack $\mathrm{MH}$, Betof A, Dearden $\mathrm{H}$, et al. Safety of resuming antiPD-1 in patients with immune-related adverse events (irAEs) during combined anti-CTLA-4 and anti-PD1 in metastatic melanoma. Ann Oncol 2018;29:250-5.

28 Simonaggio A, Michot JM, Voisin AL, et al. Evaluation of readministration of immune checkpoint inhibitors after immunerelated adverse events in patients with cancer. JAMA Oncol 2019 doi:10.1001/jamaoncol.2019.1022. [Epub ahead of print: 06 Jun 2019].

29 Li M, Sack JS, Rahma OE, et al. Outcomes after resumption of immune checkpoint inhibitor therapy after high-grade immunemediated hepatitis. Cancer 2020;126:5088-97. 